\title{
Central Opacity of Cornea
}

National Cancer Institute

\section{Source}

National Cancer Institute. Central Opacity of Cornea. NCI Thesaurus. Code C34455.

Clouding of the middle area of the cornea, usually as a result of scarring due to Infection, injury, or inflammation. 